\title{
ON THE EULER EQUATIONS OF INCOMPRESSIBLE FLUIDS
}

\author{
PETER CONSTANTIN
}

\begin{abstract}
Euler equations of incompressible fluids use and enrich many branches of mathematics, from integrable systems to geometric analysis. They present important open physical and mathematical problems. Examples include the stable statistical behavior of ill-posed free surface problems such as the Rayleigh-Taylor and Kelvin-Helmholtz instabilities. The paper describes some of the open problems related to the incompressible Euler equations, with emphasis on the blowup problem, the inviscid limit and anomalous dissipation. Some of the recent results on the quasigeostrophic model are also mentioned.
\end{abstract}

\section{INTRODUCTION}

Euler's equations for incompressible fluids, like number theory, are the wellspring of many mathematical streams. Linear partial differential equations (PDEs, henceforth), spectral theory, dynamical systems, nonlinear PDEs, geometric PDEs, harmonic analysis, completely integrable systems find in the Euler equations source, challenge and inspiration.

Euler had been involved in acoustics, hydrostatics and hydraulics research for many years by the time he wrote his treatises on fluids ([77]). After the efforts of the Bernoullis and D'Alembert, Euler's work represented a crowning manifestation of the eighteenth century's confidence in the mathematical foundations of the laws of nature. The equations are concise and capture in an idealized fashion the essence of fluid behavior.

No full account of the mathematical activity surrounding the Euler equations since their inception to this day can be attempted in a few pages. A complete description of their impact outside mathematics, from weather prediction to exploding supernovae, would fill volumes. There are books ([6], [28], [33], [105], [107]) and expository articles $([10],[42],[80])$ on the subject, too numerous to all be listed here. My own biases notwithstanding, I would like to be able to give a glimpse of some of the current activities surrounding the Euler equations and of the major directions needing further progress.

I start by casting a very wide net and describing concisely some of the areas of current research. The Euler equations cannot be separated from the surrounding scientific topics. Exposure to the broader physical context provides the mathematical researcher with both vision and power. Vision because it directs research

Received by the editors May 31, 2007.

2000 Mathematics Subject Classification. Primary 76B47; Secondary 35Q30.

Key words and phrases. Euler, anomalous dissipation, blowup, free surface, quasigeostrophic equation, vanishing viscosity, blowup, ill-posed, turbulence. 
towards fertile ground, and power because it gives the opportunity of invention of new technical tools. Therefore, in the next few pages I will present the Euler equations in connection with physical problems surrounding them.

Waves and jets and drops come to mind when thinking of fluids. These objects turn out to be mathematically the most challenging, because they involve one or two different fluids separated by an unknown ("free") surface. In the PDE community these problems go by the name of "free boundary problems". Examples include water waves, Hele-Shaw cells (oil/water interfaces in nearly two-dimensional settings), drop formation, thin films, flame fronts and more. The subject of hydrodynamic free boundary problems is vast: it includes systems that are completely integrable, nonlinear dispersive equations and stochastic models of front propagation. Imagine for instance that a very idealized description of the surface of a body of water is given by one function, $h$, the height of the water. No matter how simple, this function will have to depend at least on two variables: a position variable $x$ and a time variable $t$. The evolution in time of the function $h$ will be dictated by the Euler equations for the fluids coupled with boundary conditions for the interface. It is sometimes possible to reduce all this to an equation for $h$, expressed in terms of $h$ alone. Insisting upon such economy of the unknown comes at a price, and this equation is complicated: it is nonlinear and integro-differential (it involves simultaneously derivatives and integrals of $h$ ). Simplifications (neglecting some terms, approximating some others) lead to famous equations of water waves, such as the Korteweg de Vries equation. Murky modeling yields a pure mathematical treasure: completely integrable PDEs. Integrable PDEs are a source of significant mathematical developments that are not limited to fluid mechanics nor to PDE theory ([131]), but are outside the scope of this presentation.

Integrability in PDEs requires the ability to produce solution formulas depending on infinitely many parameters, providing the "general" solution. The well-posedness of a PDE requires the ability to solve the equation and prove the continuity of the solution with respect to initial conditions in an appropriate functional setting. It might seem that integrability would imply well-posedness, but it does not in general, and important examples of integrable free surface equations (Hele-Shaw) are ill-posed. Integrability of two-dimensional Euler equations with free surface is a current subject of research $([132])$. The modern well-posedness theory for free surfaces uses harmonic analysis and geometric PDEs ([3], [4], [35], [63], [65], [66], [102], [126], [127]) and is a subject in full swing.

Problems of hydrodynamic stability ([9], [83], [124], [129]) are of classical importance. The classical work concerns the study of the evolution of small perturbations of special, time independent solutions, usually by linearization. The linear equations obtained produce examples of nonnormal operators. The more modern theory studies the growth in time of the distance between solutions of the nonlinear equations, i.e. nonlinear instability. Instability of the solution is different from ill-posedness; actually it does not make much sense to speak of nonlinear instability of a solution without having a well-posed evolution. Ill-posedness usually means that the small scale features of the perturbation grow faster than the large scale features, and there is no cutoff in the time scales as we go to finer and finer spatial scales. This catastrophic growth prevents the continuity of the solution map in all spaces except spaces of analytic functions. Unfortunately, the distinction between 
well-posed instability and ill-posed catastrophic growth is not made in the classical physics literature, and the meanings of "instability" are multiple, including ill-posedness (in the Rayleigh-Taylor and Kelvin-Helmholtz instabilities).

Solutions of Euler equations might seem more unstable than they really are, or to be more precise, the notion of stability appropriate for them is a more generous one, that of orbital stability. An example of this nuance is the case of Kirchhoff ellipses, which are special solutions of two-dimensional Euler equations. These are ellipses that rotate at constant angular velocity, proportional to the area of the ellipse. If two ellipses have close but unequal areas, they will become, at some point in time, very different from each other. This should not imply automatically that the evolution is unstable; it just means that one should "mod-out" by the constant rotation and stay on the "leaf" defined by a fixed value of the area (which happens to be an invariant of the equation).

In the context of free surface flows, the main puzzle is a sort of "stability of instability": characteristic, predictable features arise in the physical realization of ill-posed classical instabilities such as the Rayleigh-Taylor and Kelvin-Helmholtz instabilities. For instance, characteristic plumes appear and grow in the RayleighTaylor instability (heavy fluid on top of light fluid) and generate a mixing layer that grows at a predictable rate. Also, the eye easily discerns the typical roll-up that occurs in wind-driven interfaces in the Kelvin-Helmholtz instability in clouds and waves (think of the famous Hokusai wave). Modern research established recently the elliptic nature of these ill-posed problems ([90], [97]): if solutions have a certain minimal regularity, then they are actually analytic. The "stability of instability" has yet to find a good mathematical explanation. Scaling ([11]) is the key aspect in this problem. Perhaps generalized relative entropies ([49], [110]) might be relevant for this.

Mixing and transport are subjects of great practical significance, as they relate to the diffusion of passive and active tracers, for instance pollutants in the atmosphere or plankton in the oceans. The mathematical issue is to understand the relationship between the underlying particle trajectory dynamics and macroscopic mixing properties of the flow. Classical methods of dynamical systems are relevant for the long-time effects ([118]). Strong rapid effects are studied using PDE methods ([17], [50], [55]).

When mixing and transport are studied for small scale particles, it is necessary to consider the dissipation of kinetic energy due to internal molecular processes. The Newtonian stress balance gives rise to the Navier-Stokes equations in which the dissipation of kinetic energy is represented macroscopically by the addition to the Euler equation of a Laplacian term multiplied by a positive coefficient, the kinematic viscosity. Complex fluids are fluids in which microscopic particles are suspended, altering the Newtonian stress balance and conferring new physical properties to the fluid.

The applications of complex fluids range from biology to materials science. PDE models include non-Newtonian viscoelastic models like the Oldroyd-B equations, tensor models, and kinetic models, in which Navier-Stokes equations are coupled to linear or nonlinear Fokker-Planck equations. The well-posedness theory is difficult even in two space dimensions, and consequently the mathematical theory of complex fluids is in its developing stages ([43], [48], [52], [76], [89], [98], [100], [101], [103], [115]). Some of the models of complex fluids involve stochastic PDEs, or hybrid 
systems, in which PDEs are coupled to stochastic differential equations. These are nonlinear systems with many degrees of freedom subjected to thermal noise. The mathematical theory here is also developing. The numerical analysis of these models leads naturally to questions of dimension reduction and effective algorithms that are very much in the forefront of current applied mathematical research. Stochastic models also have applications to modeling based on information theoretical ideas in geophysics and atmospheric science ([106]).

In this paper I will concentrate on a few specific problems related to the incompressible Euler equations,

$$
\partial_{t} u+u \cdot \nabla u+\nabla p=0
$$

with

$$
\nabla \cdot u=0
$$

A few words about notation: $u=\left(u_{j}\right)$ is the velocity vector with $n$ components; $u \cdot \nabla=\sum_{j=1}^{n} u_{j} \partial_{j}$ is a first order differential operator with coefficients $u_{j}$. The velocity components are functions $u_{j}=u_{j}(x, t)$ where $x \in \mathbb{R}^{n}$ and $t \in \mathbb{R}$. The notations $\partial_{t}=\frac{\partial}{\partial t}, \partial_{j}=\frac{\partial}{\partial x_{j}}$ represent partial derivatives. The equation (2) is the condition of incompressibility: the divergence $\nabla \cdot u=\sum_{j=1}^{n} \partial_{j} u_{j}$ vanishes. The scalar function $p=p(x, t)$ whose gradient $\nabla p$ appears in (1) is called the pressure. The pressure is determined up to the addition of a term that is constant in space but not necessarily in time. The equations are nonlinear, which is to say, they look nonlinear to the naked eye, with quadratic nonlinearity. (Some completely integrable equations look the same and, even more, are derived from them.) The Euler equations are also nonlocal. That means that one cannot compute the time derivative of the solution $u$ at $(x, t)$ only from knowledge of the function $u$ in a neighborhood of $x$ at time $t$. Taking the divergence of (1) we see that

$$
-\Delta p=\nabla \cdot(u \cdot \nabla u) \text {. }
$$

This is the reason for the nonlocality: the Laplacian $\Delta p$ is determined by local information about $u$, and $\nabla p$ is not.

The equations are covariant under Galilean transformations: if the coordinates move at constant speed $y=x+v t$, then the velocity in the $y$ reference frame is $u(x, t)+v$. If the coordinates are rotated by a fixed angle, then the velocities are rotated by the same angle. The equation (1) is the Eulerian description of the flow. The equations are in a sense hyperbolic: information is carried by the flow, to some extent. This is seen in the Lagrangian description (due also to Euler). We consider time dependent maps $X: \mathbb{R}^{n} \rightarrow \mathbb{R}^{n}$

$$
a \mapsto X(a, t), \quad X(a, 0)=a .
$$

These maps represent marked fluid particle trajectories; $a$ is the label of the particle. It is customary to take this label to be the position of the particle at the beginning of the observation $(t=0)$. The fact that particles travel with velocity $u$ is expressed in the system of ordinary differential equations

$$
\partial_{t} X=u(X, t)
$$

The Lagrangian formulation of (1) is Newton's second law

$$
\partial_{t}^{2} X+\left(\nabla_{x} p\right)(X, t)=0
$$


and the Lagrangian form of (2) is

$$
\operatorname{det}\left(\nabla_{a} X\right)=1 \text {. }
$$

Incompressibility refers to the fact that the maps $a \mapsto X(a, t)$ are volume preserving (in view of (6)). The divergence-free condition (2) is the Eulerian equivalent counterpart. (As is easily seen, if everything is smooth, by differentiating (4)). The Lagrangian formulation involves the Eulerian gradient of the pressure $\nabla_{x} p$ which could be computed using (3).

The system describes with great simplification the time evolution of an incompressible fluid of constant density in the absence of any forces other than those arising from the incompressibility constraint. Considering internal processes that lead to energy dissipation leads to the Navier-Stokes equations,

$$
\partial_{t} u+u \cdot \nabla u-\nu \Delta u+\nabla p=0,
$$

with the same incompressibility constraint (2). Here $\nu>0$ represents the kinematic viscosity, which is a constant (at constant temperature). The introduction of a second order operator requires (and permits) boundary conditions; these are the essential physical ingredient introduced by the presence of the Laplacian. The Navier-Stokes equations, in the presence of forces and boundary conditions, are a good description of Newtonian fluids and, when additional stresses are introduced, of non-Newtonian fluids.

\section{The BLOWUP PROBLEM}

2.1. Dimension and geometry. The blowup problem is: Can singularities arise in finite time from smooth initial velocities with finite kinetic energy?

The blowup problem for the Euler equations is a major open problem of PDE theory, of far greater physical importance than the blowup problem for the NavierStokes equation, which of course is known to nonspecialists because it is a Clay Millennium Problem. There is a deep connection between the two problems, but there are major differences as well. The blowup problem for the Euler equation concerns growth of velocity gradients of solutions and is not tamed by putting an upper bound on the velocity itself: an Euler equation singularity does not require infinite momentum, whereas a Navier-Stokes blowup does. (Interesting criteria for absence of inviscid hydrodynamic blowup based on momentum do exist, but they are for limited configurations ([64]).) The occurrence of an infinite momentum from finite smooth initial data, without external forcing, with zero or periodic boundary conditions and with positive kinematic viscosity has no physical justification. Obviously, continuum mechanics breaks down at very small scales, so there is no doubt that the equations do not hold at all scales. While this might be the case, it is not the question. The question, not to put too fine a point on it, is: do we need Schrödinger's equations to calculate the flow around a moving car? Or to predict tomorrow's weather? So far, no calculation of the purely decaying Navier-Stokes equation suggested the need for a cutoff at fixed viscosity. There are many ways one can regularize the Euler equations, and the kinematic viscosity might not be the only regularizing mechanism. On the other hand, if regularity for Navier-Stokes equations is to be proven, then the proof cannot be perturbative and new ideas are needed. Indeed, writing the Navier-Stokes equations as a perturbed heat equation is useful for small data arguments, but when the nonlinear term dominates, 
this approach ceases to be sufficient. New methods that are nonlinear, solutiondependent, need to be invented. They are to be sought in the study of the Euler equations. In fact, growth of gradients in Euler equation fashion might lead to vortex reconnection and regularity in Navier-Stokes equations ([37]). Contrary to infinite momentum singularities, production of very large gradients is physically important as it is related to the anomalous dissipation of energy, a well documented experimental fact. It is known that if there are no singularities in the solution of the Euler equations with initial data $u_{0}$ on the time interval $[0, T]$, then there can be no singularities in the Navier-Stokes solution with the same initial data and small enough viscosity ([36]). The regularity for large enough viscosities is also known. Unfortunately, there is a gap between the two ranges of viscosities, and it is not clear how to close it.

The solutions of Euler equations do not blow up from smooth, finite kinetic energy initial data when the dimension of space $n=2([8])$. When $n=3$, the problem is open. The easiest way to see the difference between the two situations is to look at the vorticity, curl of velocity $\omega=\nabla \times u$ in three dimensions. In two dimensions, $\omega=\nabla^{\perp} \cdot u$. Taking the curl of (1) we obtain the vorticity evolution equation

$$
\partial_{t} \omega+u \cdot \nabla \omega-\omega \cdot \nabla u=0 .
$$

In $n=2$ term $\omega \cdot \nabla u$ vanishes identically and the vorticity equation is a transport equation

$$
\partial_{t} \omega+u \cdot \nabla \omega=0
$$

In two dimensions it follows then that

$$
\omega(x, t)=\omega_{0}(A(x, t))
$$

where $\omega_{0}$ is the curl of the initial data and $A(x, t)=X^{-1}(x, t)$ is the "back-tolabels" map: the inverse of the Lagrangian path trajectory $a \mapsto X(a, t)$. Because the back-to-labels map preserves volume, it follows that $\|\omega(x, t)\|_{L^{p}(d x)}=$ $\left\|\omega_{0}\right\|_{L^{p}(d x)}$ holds for any $1 \leq p \leq \infty$ in $n=2$. $\left(L^{p}(d x)\right.$ is the usual Lebesgue space.) This quantitative information was extracted from the system because of a cancellation, without using quantitative information about $A(x, t)$. It is a free gift. Once the magnitude of the vorticity is controlled, persistence of smoothness follows with a little analysis.

The analogue of (10) in three dimensions is

$$
\omega(x, t)=\left(\nabla_{a} X\right)(A(x, t), t) \omega_{0}(A(x, t))
$$

which expresses the fact that the integral curves of the vorticity, the vortex lines, are carried by the flow. The two-dimensional vorticity is carried along by particle paths, its magnitude unchanged. The three-dimensional vorticity is carried as well, but its magnitude is amplified or diminished by the gradient of the flow map. If one allows for infinite kinetic energy solutions, then one can find blowup ([32], [39], [85], [86], [113], [122]). If one considers complex solutions, then again one can find blowup ([22]). Unfortunately, the infinite energy blowup also occurs in $2 \mathrm{D}$, where in fact finite energy solutions do not blow up. 
The Beale-Kato-Majda ([15]) criterion (see also ([94]) for an extension) says that the time integral of the maximum magnitude of the vorticity

$$
\int_{0}^{T}\|\omega(t)\|_{L^{\infty}(d x)} d t
$$

controls blowup or its absence. If the integral is finite and if the initial velocity is in a Sobolev space $H^{s}$ with large enough exponent $(s>5 / 2)$ or in a $C^{s}$ space with $s>1$ (and some decay in physical space, for instance $\omega_{0} \in L^{p}$ with $p>1$ ), then the solution remains smooth on the time interval $[0, T]$. Of course, if the integral is infinite, then there is finite time blowup.

The Euler equations possess local-in-time (i.e. for small $T$ ) unique smooth solutions if the initial velocity is in the spaces described above. These function spaces are Banach algebras for the gradients.

Because the magnitude of vorticity controls the blowup, it is useful to look more closely at its evolution:

$$
\left(\partial_{t}+u \cdot \nabla\right)|\omega|=\alpha|\omega|
$$

The scalar quantity $\alpha(x, t)$ is the vortex stretching factor, and obviously, by Gronwall's lemma, when $\int_{0}^{T}\|\alpha(t)\|_{L^{\infty}} d t$ is finite then the Beale-Kato-Majda criterion implies that no singularities are generated spontaneously in the time interval $[0, T]$. If $\alpha \geq c|\omega|$ holds for a long enough period of time, with $c>0$, then blowup ensues. It turns out ([37]) that $\alpha$ has a representation

$$
\alpha(x, t)=\frac{3}{4 \pi} P . V . \int_{\mathbb{R}^{3}} D(\widehat{y}, \xi(x+y, t), \xi(x, t))|\omega(x+y, t)| \frac{d y}{|y|^{3}}
$$

where

$$
D\left(e_{1}, e_{2}, e_{3}\right)=\left(e_{1} \cdot e_{3}\right) \operatorname{det}\left(e_{1}, e_{2}, e_{3}\right)
$$

and $\xi(x, t)=\frac{\omega(x, t)}{|\omega(x, t)|}$. The integral representing $\alpha$ is a principal value type integral, and consequently the dimensional analysis of the equation would predict blowup along characteristics. But the representation leads to criteria for absence of blowup ([46], [47]) that are based on the observation that if the direction field $\xi$ is regular, then the integral in (13) is not of principal value type any more, and in the case of the Navier-Stokes equations that is enough to rule out blowup. The regularity of $\xi$ in two dimensions is clear: $\xi=(0,0,1)$ in some system of coordinates. The fact $\xi$ has to have some roughness in order for blowup to be possible indicates some necessary complexity of the underlying geometric support of blowup. Moreover, single-scale self-similar behavior is impossible ([25]).

The criteria based on $\xi$ have been extended and refined ([19], [69]) and validated numerically ([87]). There exists an extensive numerical literature on the subject of finite time singularities in the Euler equations, but the problem remains undecided numerically as well.

2.2. Conserved quantities. From past experience with nonlinear PDE of physical origin, it is imperative to take advantage of conserved quantities, the "free gifts". The smooth solutions of the Euler equations conserve kinetic energy,

$$
\int_{\mathbb{R}^{3}}|u(x, t)|^{2} d x=\int\left|u_{0}(x)\right|^{2} d x
$$


helicity $([111])$

$$
\int_{\mathbb{R}^{3}} u(x, t) \cdot \omega(x, t) d x=\int_{\mathbb{R}^{3}} u_{0}(x) \cdot \omega_{0}(x) d x,
$$

and circulation

$$
\oint_{X(\gamma, t)} u(x, t) \cdot d x=\oint_{\gamma} u_{0}(a) \cdot d a .
$$

In two space dimensions, additional conservation laws are integrals of arbitrary functions of the vorticity. The circulation is the integral of the form $u \cdot d x$ on arbitrary closed loops transported by the flow. The helicity and circulation conservation laws have not been sufficiently exploited for analysis. The helicity encodes topological information about the average linking number between vortex lines. The circulation provides a connection between particle path length elements and momentum.

The conservation of energy follows easily from the form (1) of the equations. The conservation of circulation follows from the Weber formula (see ([40], [42]) and references therein)

$$
u(x, t)=\mathbb{P}\left[(\nabla A)^{*}\left(u_{0} \circ A\right)\right] .
$$

Here $\mathbb{P}$ is the Leray-Hodge projector on divergence-free functions and $(\nabla A)^{*}$ is the transposed matrix. Conservation of helicity follows also from the Weber formula. The Euler equations are expressed succinctly as a system of active scalars

$$
\partial_{t} A+u \cdot \nabla A=0
$$

with $u$ given from $A$ by (14). In this formulation, the conservation of circulation and of helicity are transparent. The Navier-Stokes equations have a deterministic parabolic formulation ([41]) that resembles (14), (15). A stochastic formulation based on (14), (15) and on seminal ideas of ([34]) was obtained as well ([88]).

2.3. Weak solutions. Because singularities cannot be ruled out, and because sometimes it makes physical sense to admit solutions with singularities in them, the notion of solution can be extended to that of weak solution. These solve a weak form of the equation in the distribution sense, placing the equations in large spaces using duality. The Euler and Navier-Stokes equations are in divergenceform, and integration by parts moves derivatives on test functions. The notions of weak solution depend on the equation and need to be handled with care.

The Navier-Stokes equations possess global weak solutions in 3D ([99]), and their singularities, if any, are confined to a space-time set of dimension less than 1 ([20]). Their uniqueness is not known.

There is no similar notion of weak solution of Euler equations in 3D. Nonuniqueness of putative weak solutions of the 3D Euler equations has been demonstrated ([67], [119], [121]). For 2D Euler there exist unique weak solutions ([130]) if the vorticity is bounded. The existence (but not the uniqueness) of weak solutions is established for $\omega \in L^{p}\left(\mathbb{R}^{2}\right)$ for $1<p<\infty$, and a framework for measure-valued solutions exists ([71], [72]). The existence of weak solutions in the limit case when the initial vorticity is a positive Radon measure with velocity locally uniformly in $L^{2}$ (positive vortex sheet data) was established in ([68]). The vortex sheet problem itself is the problem of keeping track of the evolution of the support of the measure if initially this was a smooth curve. Although there is a simple equation for 
the evolution of such a curve, the Birkhoff-Rott equation, the problem is ill-posed $([128])$.

Among the two-dimensional problems that attracted recently renewed interest is the surface quasi-geostrophic active scalar (QG) ([37], [53]). This is a model of geophysical origin, but it has been studied mathematically for more than a decade, mainly because of its similarity to the 3D Euler equations and because, as a scalar model in 2D, it is more amenable to numerical simulations then the full 3D Euler equation. The equation is deceptively simple,

$$
\partial_{t} \theta+u \cdot \nabla \theta=0
$$

with

$$
u=R^{\perp} \theta
$$

where $R=\nabla \Lambda^{-1}$ are Riesz transforms, $\Lambda=(-\Delta)^{\frac{1}{2}}$ the Zygmund operator. The analogy to the Euler equations is in the fact that the level sets of $\theta$ are like vortex lines. The analogue of the $3 \mathrm{D}$ vortex equation is the equation for $\nabla^{\perp} \theta$, a vector field that is tangent to the level sets. This vector field obeys (8) with $\omega$ replaced by $\nabla^{\perp} \theta$. Because $\theta$ is carried by the flow it creates, it is expressed in terms of the back-to-labels map as $\theta(x, t)=\theta_{0}(A(x, t))$. The analogue of (11) for $\nabla^{\perp} \theta$ holds in the same form,

$$
\left(\nabla^{\perp} \theta\right)(x, t)=\left(\nabla_{a} X\right)(A(x, t), t)\left(\nabla^{\perp} \theta_{0}\right)(A(x, t)) .
$$

There is a BKM theorem that establishes that finiteness of

$$
\int_{0}^{T}\left\|\nabla^{\perp} \theta(t)\right\|_{L^{\infty}(d x)} d t
$$

controls absence of blowup. The magnitude $\left|\nabla^{\perp} \theta\right|$ obeys the same equation as (12), and the stretching factor $\alpha$ has a principal value representation like (13) that reveals the same geometric depletion if the direction field $\xi=\nabla^{\perp} \theta /\left|\nabla^{\perp} \theta\right|$ is locally regular. Remarkably, there exist weak solutions in $L^{2}$ ([116]). There is numerical ([114]) and theoretical ([61]) evidence that no blowup occurs, but the problem is still open.

Recent new developments have been achieved in dissipative forms of the equation

$$
\partial_{t} \theta+u \cdot \nabla \theta+\kappa \Lambda^{s} \theta=0,
$$

with $\kappa>0,0<s$. If $s>1$ we say that the equation is subcritical, if $s<1$ the equation is supercritical, and if $s=1$, critical. When $s=1$ the model is physical: the dissipation represents friction with boundaries. The subcritical equations have smooth solutions ([27], [58]). In the critical case, it was known for some time that if the initial data are small in $L^{\infty}$ they remain small and the solution is regular ([44], [62]). Recently, two remarkable and quite different proofs of global existence in the critical case have been obtained. In one $([92])$ the result is one of global persistence of regularity, based on a new and promising idea, a maximum modulus of continuity principle. The other proof ([21]) uses harmonic extension to prove a gain of regularity of weak solutions, in the spirit of the De Giorgi, from $L^{2}$ to $L^{\infty}$, from $L^{\infty}$ to Hölder continuous, and beyond. Work on the supercritical case along similar lines is in progress ([59], [60]). 


\section{The VANISHING VISCOSITY LIMIT AND ANOMALOUS DISSIPATION}

3.1. Singular limits and boundary layers. The vanishing viscosity limit of solutions of Navier-Stokes equations is a singular limit (that means that the type of the equation threatens to change in the limit: the highest order derivative is multiplied by a vanishing coefficient). Singular limits are ubiquitous in applied mathematics and correspond to physical reality. In bounded domains $\Omega$, the fact that the vanishing viscosity limit is a singular limit is most vividly manifested in boundary layers. The Navier-Stokes equations are second-order differential equations and require boundary conditions, for instance homogeneous Dirichlet boundary conditions,

$$
u_{\mid \partial \Omega}=0 .
$$

For the Euler equation the only requirement is that the particle paths be tangent to the boundary

$$
u \cdot N_{\mid \partial \Omega}=0
$$

where $N$ is the normal to the boundary. In the limit of zero viscosity there are thin layers near the boundary where the change from a viscosity-dominated behavior to a nonviscous behavior takes place, or at least that is the physical picture in quiescent situations near flat boundaries. Boundary layers are very real but difficult to study rigorously, especially if the domains are curved and the flows are time-dependent ([23], [24], [74], [75]). At present the problem of the vanishing viscosity limit is open even in two space dimensions, in bounded domains. The physical problem of boundary layers, for instance in wall bounded flow, has a long history ([120]), but the classical Prandtl boundary layer theory has been challenged ([12], [13]).

More progress has been made in the study of the limit when boundaries are absent (flow in $\mathbb{R}^{n}$ or $\mathbb{T}^{n}$ ). Even in this restricted situation, there are two distinct concepts of vanishing viscosity limit. The finite-time, zero-viscosity limit is the

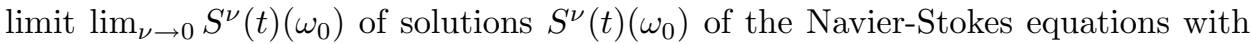
a fixed initial datum $\omega_{0}$ and with time $t$ in some finite interval $[0, T]$. By contrast, in the infinite-time, zero-viscosity limit, long-time averages of functionals of the solutions

$$
\lim _{t \rightarrow \infty} \frac{1}{t} \int_{0}^{t} \Phi\left(S^{\nu}(s) \omega_{0}\right) d s=\int \Phi(\omega) d \mu^{\nu}(\omega)
$$

are considered first, at fixed $\nu$. These are represented by measures $\mu^{\nu}$ in function space. The long-time, zero-viscosity limit is then $\lim _{\nu \rightarrow 0} \mu^{\nu}$,

$$
\lim _{\nu \rightarrow 0}\left(\lim _{t \rightarrow \infty} \frac{1}{t} \int_{0}^{t} \Phi\left(S^{\nu}(s) \omega_{0}\right) d s\right) .
$$

The two kinds of limits are not the same. This is most clearly seen in the situation of two-dimensional, unforced Navier-Stokes equations. In this case, any smooth solution of the Euler equations is a finite-time inviscid limit, but the infinite-time inviscid limit is unique: it is the function identically equal to zero. This simple example points out the fact that the infinite-time, zero-viscosity limit is more selective. In less simple situations, when the Navier-Stokes equations are forced, the long-time inviscid limit is not well understood. The finite-time, zero-viscosity limit is the limit that has been most studied. For smooth solutions in $\mathbb{R}^{3}$, the zero-viscosity limit is given by solutions of the Euler equations, for short time, in classical ([123]) and Sobolev ([91]) spaces; the limit holds for as long as the Euler solution is smooth ([36]). The convergence occurs in the Sobolev space $H^{s}$ as long 
as the solution remains in the same space $([108])$. The rates of convergence are optimal in the smooth regime, $O(\nu)$. In some nonsmooth regimes (smooth vortex patches), the finite time inviscid limit exists and optimal rates of convergence for the velocity can be obtained ([1], [108]), but the rates (for the vorticity) deteriorate when the smoothness of the initial data deteriorates - for nonsmooth vortex patches $([56],[57])$.

3.2. Anomalous dissipation. One of the most fundamental questions concerning the inviscid limit is: what happens to ideally conserved quantities?

We start by discussing this problem for two-dimensional flows where the analysis is simpler. Two-dimensional flows are relevant for atmospheric flow, which is nearly incompressible and nearly two-dimensional. In $n=2$ one of the important conserved quantities for smooth solutions of the Euler equations (one of the "free gifts") is the enstrophy,

$$
\int_{\mathbb{R}^{2}}|\omega(x, t)|^{2} d x
$$

When we solve the unforced Navier-Stokes equations and the initial vorticity is in $L^{2}$, then

and the quantity

$$
\frac{d}{2 d t} \int_{\mathbb{R}^{2}}|\omega(x, t)|^{2} d x+\nu \int_{\mathbb{R}^{2}}|\nabla \omega(x, t)|^{2} d x=0
$$

$$
\nu \int_{\mathbb{R}^{2}}|\nabla \omega(x, t)|^{2} d x
$$

is the instantaneous rate of dissipation of enstrophy. If the gradients are bounded in $L^{2}$, then this quantity vanishes as $\nu \rightarrow 0$. But of course, as $\omega$ depends on $\nu$ and as the gradients of vorticity might be large, the limit might be nonzero. The term "anomalous dissipation", favored by physicists with field-theoretical background, refers to the case when the rate of dissipation has a nonzero limit, in the zero viscosity limit. The existence of anomalous dissipation of enstrophy is essential in Kraichnan's theory of two-dimensional turbulence ([84], [95]). Bounds on the dissipation of enstrophy in physical terms were given in ([2]). Anomalous dissipation of enstrophy was studied in the framework of finite-time inviscid limits with rough initial data ([79], [104]). It was established that if the initial vorticity belongs to $L^{2}\left(\mathbb{R}^{2}\right)$, then weak solutions of the Euler equations conserve enstrophy, and that implies absence of anomalous dissipation of enstrophy for finite time.

The physical context for anomalous dissipation is, however, turbulence theory, and that refers to the long-time limit, in the presence of forces.

For long-time behavior, in the case of forced two-dimensional Navier-Stokes equations, there is a difficulty due to the inverse cascade of kinetic energy: the energy piles up at the largest scale and grows without bound. In numerical simulations and in experimental studies, energy injected at small scales is transferred to large scales (from high wave numbers to low wave numbers). Physically, this corresponds to the coalescence of small vortices into large ones. There cannot exist a steady vanishing viscosity state when the forcing does not vanish with viscosity ([96]).

Wave-number independent damping is used to model friction with boundaries and a variety of other physical mechanisms responsible for attenuating large scale turbulent energy. For example, a well-known model, the Charney-Stommel model of the Gulf Stream ([7]), is a two-dimensional, damped and driven Euler system. 
Numerical studies of two-dimensional turbulence employ devices to remove the energy that piles up at the large scales, and damping is the most common such device. D. Bernard ([18]) suggested, on the basis of field-theoretical computations, that there is no anomalous dissipation of enstrophy in damped and driven twodimensional Navier-Stokes equations.

This was recently proved rigorously in ([54]). A first result concerns sequences of time-independent individual solutions. The sequences have enough compactness to pass to convergent subsequences. The resulting solution is a weak solution of the damped and driven Euler equations. The existence of weak solutions of such equations in the case of the Charney-Stommel model was first obtained in ([7]). The weak solution of the damped and driven Euler equation is a renormalized solution in the sense of ([70]). This implies that the weak solution obeys an enstrophy balance, and that is used to show that there is no anomalous dissipation. The time-dependent situation is more complicated. Stationary statistical solutions of the damped and driven Navier-Stokes equations in the spirit of ([81], [82], [125]) need to be considered. In the case of finite-dimensional dynamical systems

$$
\frac{d \omega}{d t}=N(\omega)
$$

invariant measures $\mu$ are Borel measures in the $\omega$ phase space such that $\int \nabla_{\omega} \Psi(\omega) N(\omega) d \mu(\omega)=0$ for any test function $\Psi$. In infinite dimensions, we need to restrict the test functions to a limited class $\mathcal{T}$ of admissible functions, but the notion of a stationary statistical solution of the damped and driven Navier-Stokes equation is a natural extension of the notion of invariant measure for deterministic finite-dimensional dynamical systems.

Weak limits of stationary statistical solutions of the damped and driven NavierStokes equations are renormalized stationary statistical solutions of the damped and driven Euler equations, in the spirit of ([70]). Stationary statistical solutions $\mu^{\nu}$ of the damped and driven Navier-Stokes equations can be obtained by the KrylovBogoliubov procedure of taking long-time averages. These solutions have good enough properties so that their weak limits are renormalized stationary statistical solutions $\mu^{0}$ of the damped and driven Euler equations that obey the enstrophy balance law (expected formally from the damped and driven Euler equation by taking long-time averages). This is the central fact that allows one to prove that the zero viscosity limit of the long-time average enstrophy dissipation rate vanishes:

$$
\lim _{\nu \rightarrow 0} \nu\left(\lim \sup _{t \rightarrow \infty} \frac{1}{t} \int_{0}^{t}\left\|\nabla \omega\left(s+t_{0}\right)\right\|_{L^{2}\left(\mathbb{R}^{2}\right)}^{2} d s\right)=0
$$

holds for all solutions $\omega(t)=S^{N S, \gamma}(t)\left(\omega_{0}\right)$ of the damped and driven Navier-Stokes equation with fixed damping coefficient $\gamma$, all $t_{0}>0$, and all $\omega_{0} \in L^{p}\left(\mathbb{R}^{2}\right) \cap L^{\infty}\left(\mathbb{R}^{2}\right)$. The convergence in this class of statistical solutions is such that

$$
\lim _{\nu \rightarrow 0} \int_{L^{2}\left(\mathbb{R}^{2}\right)}\|\omega\|_{L^{2}\left(\mathbb{R}^{2}\right)}^{2} d \mu^{\nu}(\omega)=\int_{L^{2}\left(\mathbb{R}^{2}\right)}\|\omega\|_{L^{2}\left(\mathbb{R}^{2}\right)}^{2} d \mu^{0}(\omega) .
$$

The problems of anomalous dissipation in 3D are substantially more difficult. The ideally conserved quantity under investigation is the kinetic energy,

$$
\int_{\mathbb{R}^{3}}|u(x, t)|^{2} d x
$$


The kinetic energy is conserved by smooth solutions of the Euler equations. For smooth solutions of unforced Navier-Stokes equations, the kinetic energy decays,

$$
\frac{d}{2 d t} \int_{\mathbb{R}^{3}}|u(x, t)|^{2} d x+\nu \int_{\mathbb{R}^{3}}|\nabla u(x, t)|^{2} d x=0,
$$

and

$$
\nu \int_{\mathbb{R}^{3}}|\nabla u(x, t)|^{2} d x
$$

is the instantaneous rate of dissipation of kinetic energy. There is experimental and numerical evidence that the rate of dissipation of kinetic energy in Navier-Stokes equations is bounded away from zero, even at very high Reynolds numbers (small viscosities). Turbulence theory and practice is rather solidly anchored in this fact ([80], [84], [117]). The famous Kolmogorov-Obukhov ([93]) power law for the energy spectrum of turbulent fluctuations is

$$
E(k)=C \epsilon^{2 / 3} k^{-5 / 3}
$$

where $\epsilon>0$ is the nonzero rate of dissipation of kinetic energy,

$$
\epsilon=\nu\left\langle|\nabla u|^{2}\right\rangle
$$

where $\langle\ldots\rangle$ is the expected value with respect to an invariant measure supported on solutions of the Navier-Stokes equations at high Reynolds number. The fact that $\epsilon$ is bounded below, independently of viscosity, is a fundamental part of the theory. The wave number magnitude $k$ belongs to a range of values called the inertial range that extends to positive infinity in the limit of infinite Reynolds number (the vanishing viscosity limit). Independently of Kolmogorov, Onsager ([112]) suggested that there exists (in modern language) anomalous dissipation of energy and that it is supported by weak solutions of the Euler equations. Onsager conjectured that weak solutions of the Euler equation with Hölder continuity exponent $h>1 / 3$ do conserve energy and that turbulent or anomalous dissipation occurs when $h \leq 1 / 3$. The Hölder exponent 1/3 corresponds precisely to the Kolmogorov-Obukhov energy spectrum exponent $-5 / 3$. More specifically

$$
\left\langle((u(x+r e)-u(x)) \cdot e)^{p}\right\rangle \sim(\epsilon r)^{\frac{p}{3}}
$$

for $p=2$ is the Kolomogorov 2/3 law ([93]); the Kolmogorov-Obukhov energy spectrum was derived from it by dimensional analysis. In the expression above $e$ is a unit vector. Corrections to the Kolmogorov scaling for high $p$ are known in the turbulence community as "anomalous scaling". The $2 / 3$ law was challenged ([16]) and defended ([14]). Anomalous scaling has been extensively examined numerically and theoretically (but not rigorously) $([84])$, but there are competing explanations of the data $([5])$.

One direction of the Onsager conjecture was addressed mathematically ([45], [73], [78]), and it was established that energy conservation occurs for $u$ in Besov spaces $B_{3, \infty}^{s}, s>1 / 3$. Besov spaces $B_{p, q}^{s}$ are interpolation spaces, the index $s$ measures the number of derivatives, the index $p$ the $L^{p}(d x)$ space and the index $q$ is an interpolation index. Recently, the conservation of kinetic energy was proved ([29]) for velocities in $B_{3, c(\mathbb{N})}^{1 / 3}$, a space that contains $B_{3, q}^{1 / 3}$ for all $1 \leq q<\infty$, and it contains also $B_{3, \infty}^{s}$ for $s>1 / 3$. This is fairly close to optimal. There exist 
divergence-free vector fields in $B_{3, \infty}^{1 / 3}$ for which the energy flux is bounded away from zero. This implies that the instantaneous time derivative of kinetic energy of the weak solution of the Euler equations is nonzero at these fields. The existence of weak solutions of the Euler equations in $B_{3, \infty}^{1 / 3}$ has not been proved, so the counterexample does not prove the second direction of the Onsager conjecture. The proofs and examples employ the Littlewood-Paley decomposition and the flux of the Littlewood-Paley spectrum ([38]), which is a mathematically convenient variant of the physical concept of flux from the turbulence literature. For the helicity conservation, similar results apply but require more regularity (see [26] for previous sufficient conditions). If the velocity is in $B_{3, c(\mathbb{N})}^{2 / 3}$, then the helicity is conserved. There exist divergence-free velocity fields in $B_{3, \infty}^{2 / 3}$ for which the helicity flux is bounded away from zero.

Shell models are sequences of ODEs, resembling the form the Euler equation takes when written in terms of the Littlewood-Paley decomposition, but greatly simplified and truncated. A proof of one direction of the Onsager conjecture near the exponent $1 / 3$ was done in ([51]); there are models that have anomalous dissipation ([30], [31]). This can occur in linear systems as well ([109]).

It is not clear however how one would go about constructing weak solutions of the initial value problem for $3 \mathrm{D}$ Euler equations in $B_{3, \infty}^{1 / 3}$ that dissipate energy. It is possible however that the long-time, zero-viscosity limit selects very particular stationary measures supported in this space.

\section{Conclusion}

It is no exaggeration to say that the Euler equations are the very core of fluid dynamics. They use and enrich several branches of mathematics and provide important open problems. The "stability of instability" of mixing layers in ill-posed problems such as the Rayleigh-Taylor and Kelvin-Helmholtz instabilities is one such problem.

The mathematical question of blowup in the Euler equations is still one of the most challenging and meaningful problems in nonlinear PDE. The study of the vanishing viscosity limit (more properly, the infinite Reynolds number limit) in domains with boundaries is very far from completion. The problem in bounded domains is extremely challenging. The existence of weak solutions that dissipate energy by constant flux of energy, in the whole space, in the correct functional space is not known. The characterization of the zero-viscosity long-time limit statistics, including Kolmogorov-Obukhov spectrum, is open.

\section{ACKNOWLEDGMENT}

Work partially supported by NSF DMS grant No. 0504213 and by the ASC Flash Center at the University of Chicago.

\section{ABOUT THE AUTHOR}

Peter Constantin is Louis Block Professor at the University of Chicago. He was an invited speaker at the ICM in Zurich, the ICMP in Paris, and the ICIAM in Edinburgh. He is a Fellow of the Institute of Physics, London. 


\section{REFERENCES}

[1] H. Abidi, R. Danchin, Optimal bounds for the inviscid limit of Navier-Stokes equations. Asymptot. Anal. 38 (2004), 35-46. MR2060619 (2005c:35227)

[2] A. Alexakis, C. Doering, Energy and enstrophy dissipation in steady state $2 D$ turbulence. Physics Lett. A 359 (2006), 652-657.

[3] D.M. Ambrose, Well-posedness of vortex sheets with surface tension. SIAM J. Math. Anal. 35 (2003), 211-244. MR2001473 (2005g:76006)

[4] D. M. Ambrose, N. Masmoudi, The zero surface tension limit of two dimensional water waves. Commun. Pure Appl. Math. 58 (2005), 1287-1315. MR2162781 (2006d:35189)

[5] A. Arenas, A. J. Chorin, On the existence of scaling functions in turbulence according to the data. Proc. Natl. Acad. Sc. USA 103 (2006), 4352-4355. MR2213976 (2006j:76060)

[6] V. Arnold, B. Khesin, Topological methods in hydrodynamics. Springer, 1998. MR1612569 (99b:58002)

[7] V. Barcilon, P. Constantin, E. Titi, Existence of solutions to the Stommel-Charney model of the Gulf Stream. SIAM J. Math. Anal. 19 (1988), 1355-1364. MR965256 (89k:35241)

[8] C. Bardos, Existence et unicité de la solution de l'équation d'Euler en dimension deux. J. Math. Anal. Appl. 40 (1972), 769-790. MR0333488 (48:11813)

[9] C. Bardos, Y. Guo, W. Strauss, Stable and unstable ideal plane flows. Chinese Annals of Math. 23 B (2002), 149-164. MR1924132 (2003k:35192)

[10] C. Bardos, E. Titi, Euler equations of incompressible ideal fluids. Preprint 2007.

[11] G. I. Barenblatt, Scaling. CUP, Cambridge, 2003. MR2034052 (2005e:00011)

[12] G.I. Barenblatt, A.J. Chorin, Scaling laws and zero viscosity limits for wall-bounded shear flows and for local structure in developed turbulence. Comm. Pure Appl. Math. 50 (1997), 381-391. MR1438152 (98a:76041)

[13] G.I. Barenblatt, A. J. Chorin, Scaling laws and vanishing viscosity limits in turbulence theory. Proc. Symp. Appl. Math. AMS 54 (1998), 1-25. MR1492690 (99a:76065)

[14] G.I. Barenblatt, A. J. Chorin, V.M. Prostokishin, The Kolmogorov-Obukhov exponent in the inertial range of turbulence: A reexamination of experimental data. Physica D 127 (1999), 105-110. MR1677445 (2000c:76034)

[15] J.T. Beale, T. Kato, A. Majda, Remarks on the breakdown of smooth solutions for the 3-D Euler equations. Commun. Math. Phys. 94 (1984), 61-66. MR763762 (85j:35154)

[16] R. Benzi, S. Ciliberto, C. Baudet, G. Ruiz Chavarria, On the scaling of three dimensional homogeneous and isotropic turbulence. Physica D 80, 385-398 (1995). MR1312600 (95i:76046)

[17] H. Berestycki, F. Hamel, N. Nadirashvili, Elliptic eigenvalue problems with large drift and applications to nonlinear propagation phenomena. Comm. Math. Phys. 253 (2005), 451-480. MR2140256 (2006b:35057)

[18] D. Bernard, Influence of friction on the direct cascade of $2 D$ forced turbulence. Europhys. Lett. 50 (2000), 333-339.

[19] H. Beirão da Veiga, L. Berselli, On the regularizing effect of the vorticity direction in incompressible viscous flows. Diff. Int. Eqns. 15 (2002), 345-356. MR1870646 (2002k:35248)

[20] L. Caffarelli, R. Kohn, L. Nirenberg, Partial regularity of suitable weak solutions of the Navier-Stokes equations. Comm. Pure Appl. Math. 35 (1982), 771-831. MR673830 (84m:35097)

[21] L. Caffarelli, A. Vasseur, Drift diffusion equations with fractional diffusion and the quasigeostrophic equation. ArXiv: Math.AP/0608447 (2006).

[22] R. Caflisch, Singularity formation for complex solutions of the $3 D$ incompressible Euler equations. Physica D 67 (1993), 1-18. MR1234435 (94h:76013)

[23] R. Caflisch, M. Sammartino, Zero viscosity limit for analytic solutions of the Navier-Stokes equations on a half space, I. Existence for Euler and Prandtl equations. Comm. Math. Phys. 192 (1998), 433-461. MR1617542 (99d:35129a)

[24] R. Caflisch, M. Sammartino, Zero viscosity limit for analytic solutions of the Navier-Stokes equations on a half space, II. Construction of the Navier-Stokes solution. Comm. Math. Phys. 192 (1998), 463-491. MR1617538 (99d:35129b)

[25] D. Chae, Nonexistence of self-similar singularities for the 3D incompressible Euler equations. Commun. Math. Phys. (2007) (to appear).

[26] D. Chae, On the conserved quantities for the weak solutions of the Euler equations and the Quasi-Geostrophic equation. Commun. Math. Phys. 266 (2006), 197-210. MR2231970 
[27] D. Chae, On the regularity conditions for the dissipative quasi-geostrophic equations. SIAM J. Math. Anal. 37 (2006), 1649-1656. MR2215601 (2007c:76008)

[28] J-Y. Chemin, Perfect Incompressible Fluids. Clarendon Press, Oxford Univ., 1998. MR1688875 (2000a:76030)

[29] A. Cheskidov, P. Constantin, S. Friedlander, R. Shvydkoy, Energy conservation and Onsager's conjecture for the Euler equations. ArXiv; Math.AP/0704.0759 (2007).

[30] A. Cheskidov, S. Friedlander, N. Pavlović, An inviscid dyadic model of turbulence: the fixed point and Onsager's conjecture. Journal of Mathematical Physics, to appear.

[31] A. Cheskidov, S. Friedlander, N. Pavlović, An inviscid dyadic model of turbulence: the global attractor. Preprint (2007).

[32] S. Childress, G. R. Ierly, E. A. Spiegel, W. R. Young, Blow up on unsteady two-dimensional Euler and Navier-Stokes solutions having stagnation point form. J. Fluid. Mechanics 203, (1989), 1-22. MR1002875 (90e:76054)

[33] A. Chorin, J. Marsden, A mathematical introduction to fluid mechanics. 3rd edition, Springer, 1993. MR1218879 (94c:76002)

[34] A. J. Chorin, Numerical study of slightly viscous flow. J. Fluid Mech. 57 (1973), 785-796. MR0395483 (52:16280)

[35] D. Christodoulou, H. Lindblad, On the motion of the free surface of a liquid. Commun. Pure Appl. Math. 53 (2000), 1536-1602. MR1780703 (2002c:76025b)

[36] P. Constantin, Note on loss of regularity for solutions of the $3 D$ incompressible Euler and related equations. Commun. Math. Phys. 104 (1986), 311-326. MR836008 (87f:35200)

[37] P. Constantin, Geometric statistics in turbulence. SIAM Review 36 (1994), 73-98. MR1267050 (95d:76057)

[38] P. Constantin, The Littlewood-Paley spectrum in 2D turbulence. Theor. Comp. Fluid Dyn. 9 (1997), 183-189.

[39] P. Constantin, The Euler equations and nonlocal conservative Riccati equations. Intern. Math. Res. Notes 9 (2000), 455-465. MR1756944 (2001b:76007)

[40] P. Constantin, An Eulerian-Lagrangian approach for incompressible fluids: local theory. JAMS 14 (2001), 263-278. MR1815212 (2002e:76008)

[41] P. Constantin, An Eulerian-Lagrangian approach to the Navier-Stokes equations. Commun. Math. Phys. 216 (2001), 663-686. MR1815721 (2002m:76023)

[42] P. Constantin, Euler equations, Navier-Stokes equations and turbulence. In Mathematical foundation of turbulent viscous flows: Lectures given at the C.I.M.E. Summer School, Martina Franca, Italy. Editors: M. Cannone and T. Miyakawa, Springer Lecture Notes in Mathematics 1871 (2005), 1-43. MR2196360 (2007c:76001)

[43] P. Constantin, Nonlinear Fokker-Planck Navier-Stokes systems. Commun. Math. Sci. 3 (4) (2005), 531-544. MR2188682 (2007b:35249)

[44] P. Constantin, D. Cordoba, J. Wu, On the critical dissipative quasigeostrophic equation. Indiana U. Math. Journal 50 (2001) 97-107. MR1855665 (2002h:35246)

[45] P. Constantin, W. E, E. Titi, Onsager's conjecture on the energy conservation for solutions of Euler's equation. Commun. Math. Phys. 165 (1994), 207-209. MR1298949 (96e:76025)

[46] P. Constantin, C. Fefferman, Direction of vorticity and the problem of global regularity for the Navier-Stokes equations. Indiana Univ. Math. J. 42 (1993), 775. MR1254117 (95j:35169)

[47] P. Constantin, C. Fefferman, A. Majda, Geometric constraints on potentially singular solutions for the 3-D Euler equations. Commun. in PDE 21 (1996), 559-571. MR1387460 (97c:35154)

[48] P. Constantin, C. Fefferman, E. Titi, A. Zarnescu, Regularity for coupled two-dimensional nonlinear Fokker-Planck and Navier-Stokes systems. Comm. Math. Phys. (to appear, 200708). MR2276466

[49] P. Constantin, G. Iyer, Stochastic Lagrangian transport and generalized relative entropies. Commun. Math. Sci. 4 (2006), 767-777. MR2264819

[50] P. Constantin, A. Kiselev, L. Ryzhik, A. Zlatos, Diffusion and mixing in fluid flow. Annals of Math., to appear (2007).

[51] P. Constantin, B. Levant, E. Titi, Regularity of inviscid shell models of turbulence. Physical Review E 751 (2007), 016305.

[52] P. Constantin, N. Masmoudi, Global well-posedness for a Smoluchowski equation coupled with Navier-Stokes equations in 2D. Commun. Math. Phys., to appear (07-08). 
[53] P. Constantin, A. Majda, E. Tabak, Formation of strong fronts in the 2D quasi-geostrophic thermal active scalar. Nonlinearity 7 (1994), 1495-1533. MR1304437 (95i:76107)

[54] P. Constantin, F. Ramos, Inviscid limit for damped and driven incompressible Navier-Stokes equations in $\mathbb{R}^{2}$. Commun. Math. Phys., to appear (2007).

[55] P. Constantin, L. Ryzhik, A. Novikov, Relaxation in reactive flows. GAFA (2007) (to appear).

[56] P. Constantin, J. Wu, Inviscid limit for vortex patches. Nonlinearity 8 (1995), 735-742. MR1355040 (96g:76012)

[57] P. Constantin, J. Wu, The inviscid limit for non-smooth vorticity. Indiana U. Math. J. 45 (1996), 67-81. MR1406684 (97g:35129)

[58] P. Constantin, J. Wu, Behavior of solutions to 2D quasigeostrophic equations. SIAM J. Math. Anal. 30 (1999), 937-948. MR1709781 (2000j:76019)

[59] P. Constantin, J. Wu, Regularity of Hölder continuous solutions of the supercritical quasigeostrophic equation. ArXiv: Math.AP/0701592 (2007).

[60] P. Constantin, J. Wu, Hölder continuity of solutions of supercritical dissipative hydrodynamic transport equations. ArXiv: Math.AP/0701594 (2007).

[61] D. Córdoba, Nonexistence of simple hyperbolic blow-up for the quasigeostrophic equation. Annals of Math. (2) 148 (1998), 1135-1152. MR1670077 (2000j:76020)

[62] A. Córdoba, D. Córdoba, A maximum principle applied to quasi-geostrophic equations. Commun. Math. Phys. 249 (2004), 511-528. MR2084005 (2005f:76011)

[63] D. Cordoba, F. Gancedo, Contour dynamics of incompressible 3D fluids in a porous medium with different densities. Commun. Math. Phys., to appear (2007).

[64] D. Cordoba, C. Fefferman, R. de la Llave, On squirt singularities in hydrodynamics. SIAM J. Math. Analysis 36 (2004), 204-213. MR2083858 (2005i:76032)

[65] D. Coutaud, S. Shkoller, Well-posedness of the free surface incompressible Euler equations with or without surface tension. JAMS (2007).

[66] W. Craig, An existence theory for water waves in the Boussinesq and the Korteweg-de-Vries scaling limits. Commun. PDE 10 (1985), 787-1003. MR795808 (87f:35210)

[67] C. De Lellis, L. Szekelyhidi, The Euler equations as differential inclusions. Preprint (2007).

[68] J-M. Delort, Existence de nappes de tourbillon en dimension deux. JAMS 4 (1991), 553-586. MR1102579 (92f:76019)

[69] J. Deng, T. Y. Hou, X. Yu, Geometric properties and non-blowup for 3-D incompressible Euler flow. Commun. PDE 30 (2005), 225-243. MR2131052 (2006f:35215)

[70] R. DiPerna, P-L. Lions, Ordinary differential equations, transport theory and Sobolev spaces. Invent. Math. 98 (1989) 511-547. MR1022305 (90j:34004)

[71] R. DiPerna, A. Majda, Oscillations and concentrations in weak solutions of the incompressible fluid equations. Commun. Math. Phys. 108 (1987), 667-689. MR877643 (88a:35187)

[72] R. DiPerna, A. Majda, Reduced Hausdorff dimension and concentration-cancellation for two dimensional incompressible flow. JAMS 1 (1988), 59-95. MR924702 (89e:35126)

[73] J. Duchon, R. Robert, Inertial energy dissipation for weak solutions of incompressible Euler and Navier-Stokes equations. Nonlinearity 13 (2000), 249-255. MR1734632 (2001c:76032)

[74] W. E, Boundary layer theory and the zero viscosity limit of the Navier-Stokes equation. Acta Math. Sinica (Engl. ser.) 16 (2000), 207-218. MR1778702 (2001d:76037)

[75] W. E, B. Enquist, Blow up of solutions to the unsteady Prandtl equation. Comm. Pure Appl. Math. 50 (1997), 1287-1293. MR1476316 (99c:35196)

[76] W. E, T. Li, P. Zhang. Well-posedness for the dumbbell model of polymeric fluids. Comm. Math. Phys. 248(2) (2004), 409-427. MR2073140 (2005d:35210)

[77] L. Euler, Principes généraux du mouvement des fluides. Mémoires de L'Académie Royale des Sciences et des Belles-Lettres de Berlin 11 (4 September 1755, printed 1757), 217-273; reprinted in Opera Omnia ser. 2 12, 219-250.

[78] G. L. Eyink, Energy dissipation without viscosity in ideal hydrodynamics. I. Fourier analysis and local energy transfer. Phys. D 78 (1994), 222-240. MR1302409 (95m:76020)

[79] G. Eyink, Dissipation in turbulent solutions of 2D Euler equations. Nonlinearity 14 (2001), 787-802. MR1837638 (2002g:76070)

[80] G. L. Eyink, K. R. Sreenivasan, Onsager and the theory of hydrodynamic turbulence. Rev. Mod. Phys. 78 (2006), 87-135. MR2214822 (2007g:76108)

[81] C. Foiaş, Statistical study of the Navier-Stokes equations I. Rend. Sem. Mat. Univ. Padova 48 (1972), 219-348. MR0352733 (50:5220) 
[82] C. Foiaş, Statistical study of the Navier-Stokes equations II. Rend. Sem. Mat. Univ. Padova 49 (1973), 9-123. MR0352733 (50:5220)

[83] S. Friedlander, A. Lipton-Lifschitz, Localized instabilities in fluids. Handbook of mathematical fluid dynamics, Vol. II, North-Holland, Amsterdam (2003), 289-354. MR1984155 (2004g:76072)

[84] U. Frisch, Turbulence. The legacy of A. N. Kolmogorov. Cambridge University Press, Cambridge, 1995. MR1428905 (98e:76002)

[85] J.D. Gibbon, D.R. Moore, J.T. Stuart, Exact, infinite energy blow-up solutions of the threedimensional Euler equations. Nonlinearity 16 (2003), 1823-1831. MR1999581 (2004j:35232)

[86] J. D. Gibbon, A. Fokas, C.R. Doering, Dynamically stretched vortices as solutions of the 3 D Navier-Stokes equations. Physics D 132 (1999), 497-510. MR1704825 (2000h:76047)

[87] T. Y. Hou, R. Li, Dynamic depletion of vortex stretching and non-blow up of the 3-D incompressible Euler equations. Nonlinear Science 16 (2006), 639-664. MR2271429 (2007f:76014)

[88] G. Iyer, A stochastic Lagrangian formulation of the incompressible Navier-Stokes and related transport equations. PhD Thesis, The University of Chicago (2006).

[89] B. Jourdain, T. Lelièvre, C. Le Bris. Existence of solution for a micro-macro model of polymeric fluid: the FENE model. J. Funct. Anal. 209(1) (2004), 162-193. MR2039220 (2005a:76006)

[90] V. Kamotski, G. Lebeau, On the 2D Rayleigh-Taylor instabilities. Asymptot. Anal. 42 (2005), 1-27. MR2133872 (2006b:76038)

[91] T. Kato, Nonstationary flows of viscous and ideal fluids in $\mathbf{R}^{3}$. J. Funct. Anal. 9 (1972), 296-305. MR0481652 (58:1753)

[92] A. Kiselev, F. Nazarov, A. Volberg, Global well-posedness for the critical 2D dissipative quasi-geostrophic equation. Invent. Math. 167 (2007), 445-453. MR2276260

[93] A. N. Kolmogorov, The local structure of turbulence in incompressible viscous fluids at very large Reynolds numbers. Dokl. Akad. Nauk. SSSR 30 (1941), 301-305. MR0004146 (2:327d)

[94] H. Kozono, Y. Taniuchi, Limiting case of the Sobolev inequality in BMO, with application to the Euler equations. Commun. Math. Phys. 214 (2000), 191-200. MR1794270 (2002k:46081)

[95] R. H. Kraichnan, Inertial ranges in two-dimensional turbulence. Phys. Fluids 10 (1967), 1417-1423.

[96] S. Kuksin, A. Shirikyan, Some limiting properties of randomly forced 2D Navier-Stokes equations. Proc. Roy. Soc. Edinburgh sect. A 133 (2003), 875-891. MR2006207 (2005c:60079)

[97] G. Lebeau, Régularité du problème de Kelvin-Helmholtz pour l'équation d'Euler $2 D$. ESAIM:COCV 8 (2002), 801-825. MR1932974 (2004a:76011)

[98] C. Le Bris, P-L. Lions, Existence and uniqueness of solutions to Fokker-Planck type equations with irregular coefficients. Rapport de receherche du CEREMICS 349, April 2007.

[99] J. Leray, Essai sur le mouvement d'un liquide visqueux emplissant l'espace. Acta Mathematica 63 (1934), 193-248. MR1555394

[100] F.-H. Lin, C. Liu, P. Zhang. On hydrodynamics of viscoelastic fluids. Comm. Pure Appl. Math. 58(11) (2005), 1437-1471. MR2165379 (2006d:76005)

[101] F.-H. Lin, P. Zhang, Z. Zhang. On the global existence of smooth solution to the 2-d FENE dumbbell model. Preprint, 2007.

[102] H. Linblad, Well-posedness for the motion of an incompressible liquid with free surface boundary. Annals of Math. (2) 162 (2005), 109-194. MR2178961 (2006g:35293)

[103] P.-L. Lions, N. Masmoudi. Global existence of weak solutions to micro-macro models. C. R. Math. Acad. Sci. Paris, 2007.

[104] M. Lopes Filho, A. Mazzucato, H. Nussenzveig-Lopes, Weak solutions, renormalized solutions and enstrophy defects in 2D turbulence. ARMA 179 (2006), 353-387. MR2208320 (2006k:35234)

[105] A. Majda, A. Bertozzi, Vorticity and incompressible flow. CUP, Cambridge, 2002. MR1867882 (2003a:76002)

[106] A. Majda, X. Wang, Nonlinear dynamics and statistical theories for basic geophysical flows. CUP, Cambridge (2006).

[107] C. Marchioro, M. Pulvirenti, Mathematical theory of incompressible nonviscous fluids. Applied Mathematical Sciences, 96, Springer, 1994. MR1245492 (94k:76001)

[108] N. Masmoudi, Remarks about the inviscid limit of the Navier-Stokes system. Comm. Math. Phys. 270 (2007), 777-788. MR2276465 
[109] J. Mattingly, T. Suidan, E. Vanden-Eijnden, Simple systems with anomalous dissipation and energy cascade. Commun. Math. Phys., to appear 2007-08.

[110] P. Michel, S. Mischler, B. Perthame, General entropy equations for structured population models and scattering. C.R. Acad. Sci. Paris Ser. I 338 (2004), 697-702. MR2065377 (2005b:35019)

[111] H. K. Moffatt, A. Tsinober, Helicity in laminar and turbulent flow. Ann. Rev. Fluid Mech. 24 (1992), 281-312. MR1145012 (93b:76018)

[112] L. Onsager, Statistical hydrodynamics. Nuovo Cimento (Supplemento) 6 (1949), 279-287. MR0036116 (12:60f)

[113] K. Ohkitani, J.D. Gibbon, Numerical study of singularity formation in a class of Euler and Navier-Stokes flows. Physics of Fluids 12 (2000), 3181-3194. MR1796376 (2001i:76008)

[114] K. Ohkitani, M. Yamada, Inviscid and inviscid-limit behavior of a surface quasi-geostrophic flow. Phys. Fluids 9 (1997), 876-882. MR1437554 (97m:76032)

[115] F. Otto, A.E. Tzavaras, Continuity of velocity gradients in suspensions of rod-like molecules. SFB preprint Nr. 141 (2004).

[116] S. Resnick, Dynamical problems in nonlinear advective partial differential equations. Ph.D. Thesis, University of Chicago, 1995.

[117] R. Robert, Statistical Hydrodynamics (Onsager revisited). Handbook of Mathematical Fluid Dynamics, vol. 2 (2003), 1-55. Eds. Friedlander and Serre. Elsevier. MR1983588 (2005a:76004)

[118] V. Rom-Kedar, A. Leonard, S. Wiggins, An analytical study of transport, mixing and chaos in unsteady vortical flow. J. Fluid Mech. 214 (1990), 347-394. MR1054106 (91d:76017)

[119] V. Scheffer, An inviscid flow with compact support in space-time. J. Geom. Anal. 3(4) (1993), 343-401. MR1231007 (94h:35215)

[120] H. Schlichting, K. Gersten, E. Krause, H. Oertel, C. Mayes, Boundary-Layer Theory. 8th edition, Springer, 2000. MR1765242 (2001c:76039)

[121] A. Shnirelman, On the nonuniqueness of weak solution of the Euler equation. Comm. Pure Appl. Math. 50 (1997), 1261-1286. MR1476315 (98j:35149)

[122] J. T. Stuart, Nonlinear Euler partial differential equations: singularities in their solution. In Applied Mathematics, Fluid Mechanics, Astrophysics (Cambridge, MA, 1987), World. Scien., Singapore (1988), 81-95. MR973917

[123] H.S.G. Swann, The convergence with vanishing viscosity of nonstationary Navier-Stokes flow to ideal flow in $\mathbf{R}^{3}$. Trans. Amer. Math. Soc. 157 (1971), 698-726. MR0277929 $(43: 3662)$

[124] M. Vishik, Spectrum of small oscillations of an ideal fluid and Lyapunov exponents. J. Math. Pures Appl. (9) 75 (1996), 531-557. MR1423046 (97k:35203)

[125] M. Vishik, A. Fursikov, Mathematical problems of statistical hydrodynamics. Kluwer AP, Dordrecht (1988).

[126] S. Wu, Well-posedness in Sobolev spaces of the full water wave problem in 2-D. Invent. Math. 130 (1997), 39-72. MR1471885 (98m:35167)

[127] S. Wu, Well-posedness in Sobolev spaces of the full water wave problem in 3-D. JAMS 12 (1999), 445-495. MR1641609 (2001m:76019)

[128] S. Wu, Mathematical analysis of vortex sheets. Commun. Pure Appl. Math. 59 (2006), 1065-1206. MR2230845

[129] V. I. Yudovich, The linearization method in hydrodynamical stability theory. Translations of Mathematical Monographs, 74, AMS, Providence, RI (1989) (Translated from Russian by J.R. Schulenberger). MR1003607 (90h:76001)

[130] V. Yudovich, Non-stationary flow of an ideal incompressible liquid. Zh. Vych. Mat. Mat. Fiz. 3 (1963), 1032-1066. MR0158189 (28:1415)

[131] V. Zakharov (editor), What Is Integrability? Springer Series in Nonlinear Dynamics, Springer (1991). MR1098334 (91k:58005)

[132] V. Zakharov, talk at the University of Chicago, May 2007.

Department of Mathematics, University of Chicago, Chicago, Illinois 60637

E-mail address: const@math.uchicago.edu 